\title{
Primary gastric natural killer/T-cell lymphoma with diffuse CD30 expression and without CD56 expression: A case report
}

\author{
LIZHI ZHANG ${ }^{1}$, PENGXIN ZHANG $^{1}$, JINHUI WEN ${ }^{2}$, XUEHUA CHEN $^{2}$ and HUA ZHANG ${ }^{2}$ \\ Departments of ${ }^{1}$ Pathology and ${ }^{2}$ Geratology, First Affiliated Hospital of Dalian Medical University, \\ Dalian, Liaoning 116011, P.R. China
}

Received January 7, 2015; Accepted November 20, 2015

DOI: $10.3892 / \mathrm{ol} .2015 .4015$

\begin{abstract}
The majority of natural killer (NK)/T-cell lymphomas occur in the nasal cavity and rarely involve the stomach. They possess a specific immunophenotype, with the expression of cluster of differentiation (CD)56, CD2 and CD3 $\varepsilon$, but without CD3 expression. Few cases of NK/T-cell lymphoma have partial CD30 expression. The present study reveals a unique case of a 41-year-old female patient with gastric NK/T-cell lymphoma that did not express CD56 and diffusely expressed CD30. Immunohistochemical staining demonstrated that the tumor cells expressed CD3e, CD43, CD30 and granzyme B and did not express CD2, CD4, CD5, CD7, CD8, CD56, anaplastic lymphoma kinase, CD20, paired box- 5 or pan cytokeratin. Based on the immunostaining profile and morphological features, the initial diagnosis considered was gastric anaplastic large cell lymphoma. However, following a consultation with other pathologists, the Epstein-Barr virus (EBV) status of the patient was investigated to exclude a diagnosis of NK/T-cell lymphoma. Notably, the signal for EBV RNA was diffuse positive. Therefore, the final diagnosis was corrected to NK/T-cell lymphoma. To the best of our knowledge, the present study is the first to report NK/T-cell lymphoma in the stomach with a diffuse CD30-positive and CD56-negative phenotype.
\end{abstract}

\section{Introduction}

Extranodal natural killer (NK)/T-cell lymphoma is markedly associated with Epstein-Barr virus (EBV) and is clinically aggressive, with a poor prognosis. This type of lymphoma is predominantly located in the nasal cavity and paranasal sinus, but rarely occurs in the stomach (1). The specific immunophenotype demonstrates the expression of CD56, CD2, CD3e

Correspondence to: Professor Hua Zhang, Department of Geratology, First Affiliated Hospital of Dalian Medical University, 222 Zhongshan Road, Xigang District, Dalian, Liaoning 116011, P.R. China

E-mail: zhanghua2233@126.com

Key words: NK/T-cell lymphoma, stomach, CD56, CD30 and cytotoxic granule-associated protein, diffuse expression of EBV-encoded RNA (EBER), and no expression of surface $\mathrm{CD}^{-}$(2). In the majority of patients with NK/T-cell lymphoma, CD56 is expressed and CD30 is not expressed or partially expressed, but the absence of CD56 expression and presence of CD30 expression is rare (2). The expression of CD30 is also a characteristic of cutaneous T-cell lymphoma or anaplastic large T-cell lymphoma (ALCL) (3). Therefore, the overlap of immunohistochemical phenotypes between different tumors may occasionally result in a misdiagnosis. To the best of our knowledge, no study has reported gastric NK/T-cell lymphoma without CD56 expression and with diffuse CD30 expression. It is extremely important to differentiate NK/T-cell lymphoma from other gastric neoplasms, including poorly-differentiated adenocarcinoma, ALCL, NK-cell lymphoproliferative lymphomatoid gastropathy and enteropathy-associated T-cell lymphoma (EATL) type I.

\section{Case report}

A 41-year-old woman presented to the First Affiliated Hospital of Dalian Medical University (Dalian, Liaoning, China) in January 2013, with a history of various clinical symptoms for 2 months, including epigastric pain and abdominal distention, which had been ongoing for two months. An endoscopic examination revealed an ulcer located in the cardia of the stomach, which was $4.0 \times 3.5 \mathrm{~cm}$ in size (Fig. 1A and B). Following an endoscopic biopsy, an initial diagnosis of a poorly-differentiated adenocarcinoma was made. Subsequently, a total gastrectomy with regional lymph node dissection was performed. The resected ulcer was irregular in shape with raised edges and was covered with pale yellow exudates. The cut face of the ulcer was gray and solid, and the tumor infiltrated the whole wall of the stomach.

A microscopic examination of the gastric ulcerative lesion revealed that the majority of the surface was overlaid with necrosis and the whole layer was infiltrated with discohesive and monomorphous atypical cells (Fig. 2A and B). The tumor cells were large with an irregular kidney shape and possessed dense or vesicular nuclei with abundant eosinophilic cytoplasm (Fig. 2C). Neutrophils and eosinophils were observed at the periphery of the tumor. Minor coagulative necrosis was evident (Fig. 2D). Angiocentric and angiodestructive growth patterns and large nucleoli were not observed. 

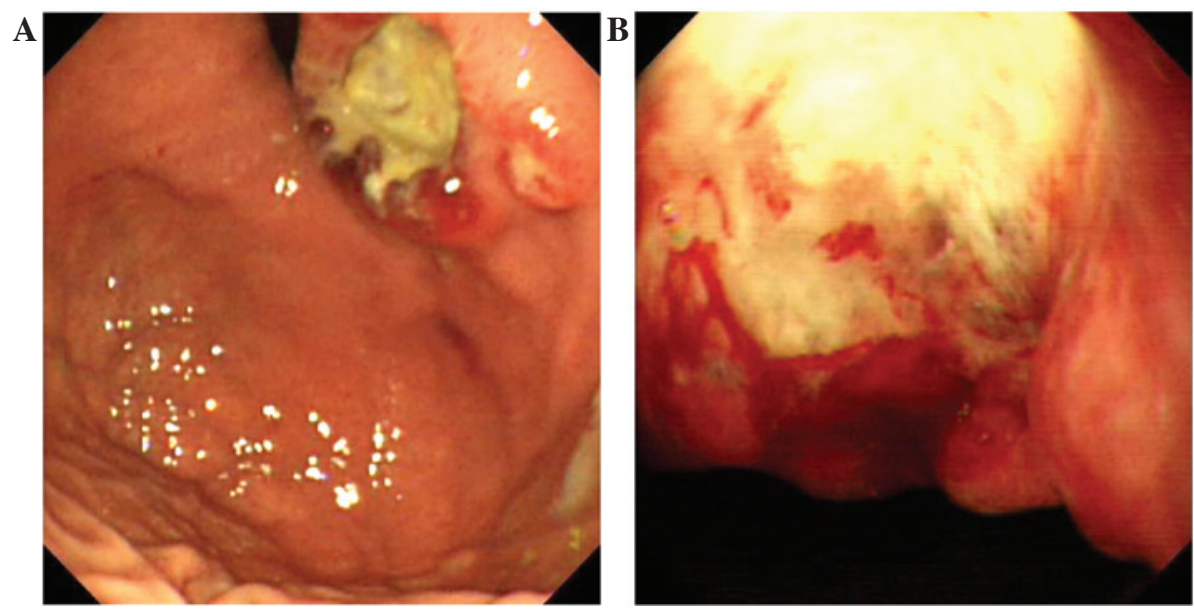

Figure 1. Endoscopic features. (A) The ulcerative lesions were mainly observed in the cardia of the stomach. (B) The ulcer was covered with pale yellow exudates.
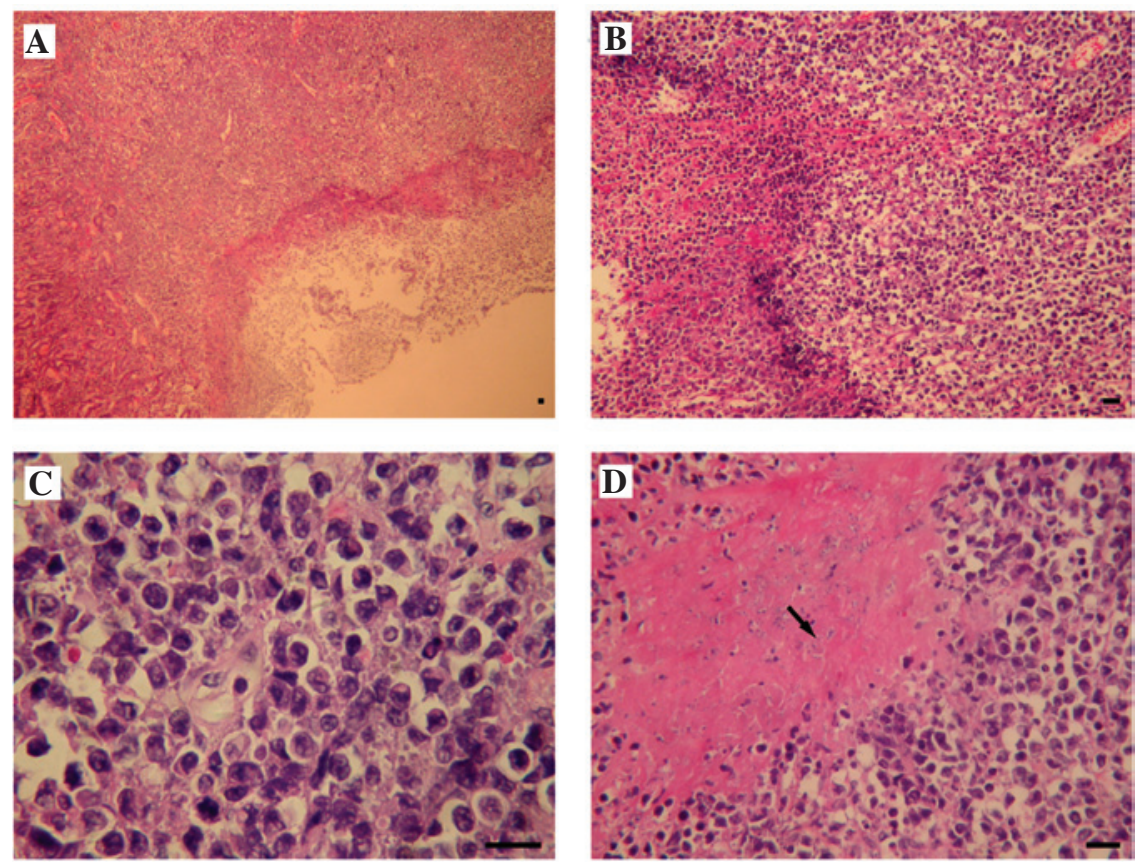

Figure 2. Histological characteristics revealed using hematoxylin and eosin staining. (A) The mucosal architecture was replaced by an ulcerative tumor, the gastric mucosa is exhibited on the left and the tumor on the right. (B) There was an overlay of necrosis on the surface of the ulcerative lesion. (C) The tumor cells were large with irregular kidney-shaped, dense or vesicular nuclei and abundant eosinophilic cytoplasms. (D) The tumor exhibited coagulative necrosis, as indicated by the black arrow. Scale bar, $20 \mu \mathrm{m}$. Magnification, x200.

Immunohistochemical staining demonstrated that the monomorphous tumor cells demonstrated diffuse expression of CD3e, CD30, granzyme-B and CD43 (Fig. 3A-D). The tumor cells did not express CD56 (Fig. 3E), cytokeratin (Fig. 3F), CD2, CD4, CD5, CD7, CD8, anaplastic lymphoma kinase, CD20 or paired box-5 transcription factor (PAX5) (data not shown). All the antibody in the immunohistochemical staining are bought from Fuzhou Maixin Biotech Co., Ltd., Fuzhou, China). The Ki-67 (ZhongShanJinQiao Co., Ltd., Beijing, China) labeling index was $80 \%$ (Fig. 3G).

Based on the morphology and immunophenotype, the diagnosis of the tumor was altered to ALCL. Following a consultation with other pathologists, the detection of EBV was advised to elimate the possibility that the tumor was a NK/T-cell lymphoma. Consequently, the tumor cells were analyzed for EBER status by in situ hybridization. As a result, the EBV RNA signal was identified as diffuse positive (Fig. 3H). Therefore, the final diagnosis was corrected to primary gastric NK/T-cell lymphoma. Additional laboratory examinations, including a hematological examination, of the patient were normal. Levels of tumor markers were within normal limits, as follows: Carcinoembryonic antigen (CEA), $0.432 \mathrm{ng} / \mathrm{ml}$ (normal, $<5.000 \mathrm{ng} /$ $\mathrm{ml}$ ); carbohydrate antigen (CA) 19-9, $12.26 \mathrm{U} / \mathrm{ml}$ (normal, $<27.00 \mathrm{U} / \mathrm{ml}$ ) and CA125, $21.0 \mathrm{U} / \mathrm{ml}$ (normal, $<35.0 \mathrm{U} / \mathrm{ml}$ ). The patient did not possessed nasal cavity lesions, and no superficial lymphadenopathy or hepatosplenomegaly was observed. Following chemotherapy, the patient was alive without tumor recurrence subsequent to a 14-month follow-up period. 

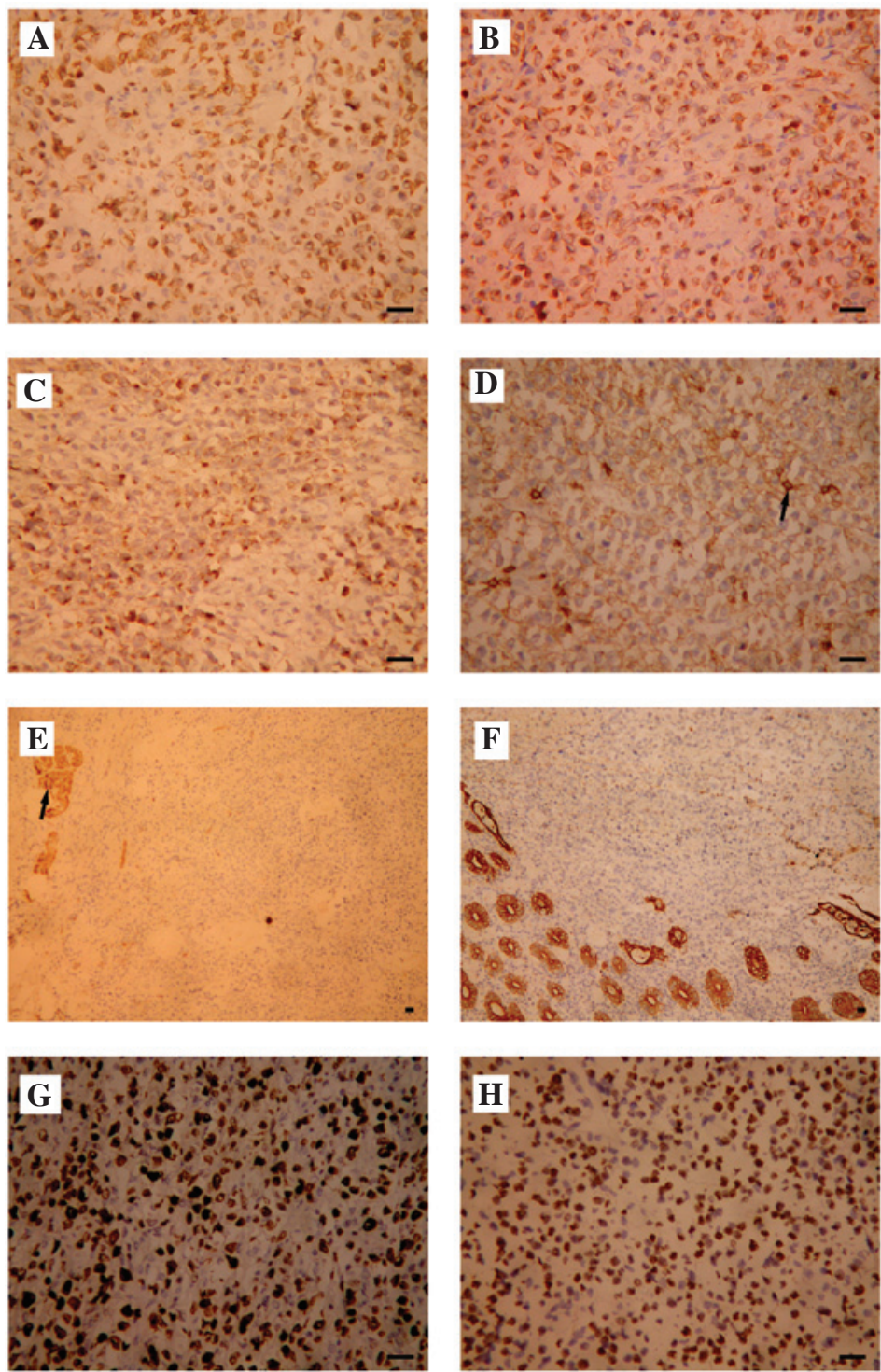

Figure 3. Immunohistochemical staining and in situ hybridization. The expression profiles of different phenotypic markers in tumor cells were observed using immunostaining, as follows: (A) CD3e, diffusely expressed; (B) CD30, diffusely expressed in the membrane and golgi; (C) granzyme-B, expressed; (D) CD43, expressed, the black arrow indicates marked expression of normal reactive T cells; (E) CD56, not expressed, the black arrow indicates residual nerve fibers that express CD56, which acts as an internal control to validate the CD56 negative staining of tumor cells; (F) cytokeratin AE1/AE3, expressed in the glandular epithelium; (G) Ki-67 index, 80\%; and (H) EBV RNA, diffusely expressed, as detected by EBV-encoded RNA in situ hybridization. Scale bar=20 $\mu$ m. x200, magnification. $\mathrm{CD}$, cluster of differentiation; EBV, Epstein-Barr virus.

The present study was approved by the Ethics Committee of The First Affiliated Hospital of Dalian Medical University. Written informed consent was obtained from the patient for the publication of the present study and the use of the accompanying images.

\section{Discussion}

NK/T-cell lymphoma may overlap in morphology, results of endoscopic imaging and immunophenotype with numerous other tumors of the stomach. Therefore, careful consideration is required to distinguish NK/T-cell lymphoma from other tumors of the stomach, and this may be based on pathological and phenotypic features.

Poorly-differentiated adenocarcinoma, which is the most common tumor of the stomach, is the primary choice for a differential diagnosis of a stomach tumor (4). The results of endoscopic imaging and the morphological features of a poorly-differentiated adenocarcinoma occasionally resemble NK/T-cell lymphoma (5). In the present study, the tumor was removed using an endoscopic biopsy and was diagnosed as poorly-differentiated adenocarcinoma, based on the tumor morphology. However, the adenocarcinoma did not express leukocyte common antigen (LCA), a T cell and B cell marker, and did express cytokeratin. Therefore, the present study recommends that when a tumor diagnosis of poorly differentiated adenocarcinoma is considered, immunostaining for cytokeratin and LCA should be performed to exclude a diagnosis of lymphoma.

ALCL is the secondary choice for a differential diagnosis in tumors of the stomach that express CD30 (6). In the present study, the tumor cells possessed irregular kidney-shaped nuclei, which expressed $\mathrm{CD} 3 \varepsilon$ and diffusely expressed CD 30 , but did not express CD56, therefore leading to an initial diagnosis of 
ALCL. However, there have been studies reporting NK/T-cell lymphoma in the nasal cavity, prostate, spleen and adrenal glands, where the tumor expresses CD30, with or without CD56 expression (7-10). To the best of our knowledge, no studies have reported this type of lymphoma in the stomach. The previous studies concluded that the absence of a CD56 signal is not sufficient to exclude NK/T-cell lymphoma, and therefore, EBV detection is required $(5,6,11,12)$. The present study observed that the tumor cells did not have clear nucleoli and demonstrated clear coagulative necrosis, which does not support a diagnosis of ALCL. However, the diagnosis of the patient was only altered when the EBV status of the patient was assessed. The patient expressed EBV RNA, leading to a final diagnosis of NK/T-cell lymphoma.

NK-cell lymphoproliferative lymphomatoid gastropathy should also be considered since this type of tumor expresses CD3 and CD56, therefore resembling NK/T-cell lymphoma. However, NK-cell lymphoproliferative lymphomatoid gastropathy has a low proliferative index (Ki-67) and an indolent clinical course with an improved prognosis. Notably, this disease is not associated with an EBV infection $(13,14)$.

EATL type I is often observed in conjunction with refractory celiac disease and mainly occurs in the jejunum and ileum, but rarely occurs in the stomach. This lymphoma should also be distinguished from gastric NK/T-cell lymphoma. EATL type I possesses large or pleomorphic cells that are express CD3, partially express CD30, but usually do not express CD8 and CD56. EATL also lacks EBV RNA expression in an EBER in situ hybridization (15).

Mucosa-associated lymphoid tissue lymphoma and diffuse large B-cell lymphoma are other common types of lymphomas that occur in the stomach, where atypical lymphoid cells diffusely infiltrate the gastric mucosa. Furthermore, the B cell phenotype is characterized by the expression of CD20 and PAX5 $(16,17)$. Consequently, these lymphomas may be easily distinguished from NK/T-cell lymphoma.

NK/T-cell lymphomas that express CD30, with or without CD56 expression, have been reported in previous studies in the nasal cavity, spleen, prostate and adrenal glands (7-10,18-20). However, to the best of our knowledge, no studies have reported this NK/T-cell lymphoma phenotype occurring in the stomach. The present study reports a unique case of primary gastric NK/T-cell lymphoma without CD56 expression and with diffuse CD30 expression in a 41-year-old woman. Due to the occurrence of this rare phenotype of NK/T-cell lymphoma, the present study recommends that the EBV status of a patient should be investigated to avoid a misdiagnosis.

\section{References}

1. Kobold S, Merz H, Tiemann M, Mahuad C, Bokemeyer C, Koop I and Fiedler W: Primary NK/T cell lymphoma nasal type of the stomach with skin involvement: A case report. Rare Tumors 1: e58, 2009.

2. Chan JKC, Quintanilla-Martinez L, Ferry JA and Peh SC: Extranodal NK/T-cell lymphoma, nasal type. In: World Health Organization Classification of Tumours of Haematopoietic and Lymphoid Tissues. Swerdlow SH, Campo E, Harris NL, Jaffe ES Pileri SA, Stein H, Thiele J and Vardiman JW (eds). Vol. 2. 4th edition. IARC Press, Lyon pp285-288, 2008.
3. de Leval L and Gaulard P: Pathology and biology of peripheral T-cell lymphomas. Histopathology 58: 49-68, 2011.

4. Espejo Romero H, Navarrete Siancas J: Classification of stomach adenocarcinomas. Rev Gastroenterol Peru 23(3):199-212, 2003.

5. Kim H, Lee D, Kim JW, Huh J, Park SH, Ha HK, Suh C, Yoon SM, Kim KJ, Choi KD, et al: Endoscopic and clinical analysis of primary T-cell lymphoma of the gastrointestinal tract according to pathological subtype. J Gastroenterol Hepatol 29: 934-943, 2014.

6. Wang FH, Li YH, Zeng J, Rao HL, Xia ZJ, Sun XF, Huang HQ, Lin TY, Jiang WQ and Guan ZZ: Clinical analysis of primary systemic anaplastic large cell lymphoma: A report of 57 cases. Ai Zheng 28: 49-53, 2009.

7. Tsukahara T, Takasawa A, Murata M, Okumura K, Nakayama M, Sato N and Hasegawa T: NK/T-cell lymphoma of bilateral adrenal glands in a patient with pyothorax. Diagn Pathol 7: 114, 2012.

8. Cao Q, Huang Y, Ye Z, Liu N, Li S and Peng T: Primary spleen extranodal NK/T cell lymphoma, nasal type, with bone marrow involvement and CD30 positive expression: A case report and literature review. Diagn Pathol 9: 169, 2014.

9. Ferenczi K, Summers P, Aubert P, Cooper B, Meyerson H, Cooper KD and Honda K: A case of CD30+ nasal natural killer/T-cell lymphoma. Am J Dermatopathol 30: 567-571, 2008.

10. Jiang Q, Liu S, Peng J, Xiong H, Xiong Z, Yang Y, Tan X and Gao X: An extraordinary T/NK lymphoma, nasal type, occurring primarily in the prostate gland with unusual CD30 positivity: Case report and review of the literature. Diagn Pathol 8: 94, 2013.

11. Bautista-Quach MA, Ake CD, Chen $M$ and Wang $J$ : Gastrointestinal lymphomas: Morphology, immunophenotype and molecular features. J Gastrointest Oncol 3: 209-225, 2012.

12. Kawamoto K, Nakamura S, Iwashita A, Watanabe J, Oshiro Y, Nakayama Y, Nimura S, Kimura N, Aoyagi K, Yao T, et al: Clinicopathological characteristics of primary gastric T-cell lymphoma. Histopathology 55: 641-653, 2009.

13. Mansoor A, Pittaluga S, Beck PL, Wilson WH, Ferry JA and Jaffe ES: NK-cell enteropathy: a benign NK-cell lymphoproliferative disease mimicking intestinal lymphoma: clinicopathologic features and follow-up in a unique case series. Blood 117: 1447-1452, 2011.

14. Tanaka T, Megahed N, Takata K, Asano N, Niwa Y, Hirooka Y and Goto H: A case of lymphomatoid gastropathy: An indolent CD56-positive atypical gastric lymphoid proliferation, mimicking aggressive NK/T cell lymphomas. Pathol Res Pract 207: 786-789, 2011 .

15. Wang L, Liu Y, Lin XY, Yu JH, Miao Y, Qiu XS and Wang EH: A case of enteropathy-associated T-cell lymphoma (type I) arising in stomach without refractory celiac disease. Diagn Pathol 7: 172, 2012.

16. Ferreri AJ and Montalbán C: Primary diffuse large B-cell lymphoma of the stomach. Crit Rev Oncol Hematol 63: 65-71, 2007.

17. Psyrri A, Papageorgiou S and Economopoulos T: Primary extranodal lymphomas of stomach: Clinical presentation, diagnostic pitfalls and management. Ann Oncol 19: 1992-1999, 2008.

18. Chang BH, Stork L and Fan G: A unique case of adolescent CD56-negative extranodal NK/T-cell lymphoma, nasal type. Pediatr Dev Pathol 11: 50-54, 2008.

19. Pongpruttipan T, Kummalue T, Bedavanija A, Khuhapinant A, Ohshima K, Arakawa F, Niino D and Sukpanichnant S: Aberrant antigenic expression in extranodal NK/T-cell lymphoma: A multi-parameter study from Thailand. Diagn Pathol 6: 79, 2011.

20. Schwartz EJ, Molina-Kirsch H, Zhao S, Marinelli RJ, Warnke RA and Natkunam Y: Immunohistochemical characterization of nasal-type extranodal NK/T-cell lymphoma using a tissue microarray: An analysis of 84 cases. Am J Clin Pathol 130: 343-351, 2008. 